\title{
Evidence for genetic anticipation in nodal osteoarthritis
}

\author{
Gary D Wright, Marian Regan, Chris M Deighton, Gillian Wallis, Michael Doherty
}

\begin{abstract}
Objective-Evidence was sought for genetic anticipation (disease occurring at an earlier age in subsequent generations, with increasing severity) in nodal osteoarthritis (NOA).

Methods-Age at symptom onset and disease severity was compared within 30 parent/offspring pairs with NOA. Correlation between the offspring age of disease onset and the parental age at conception was also assessed.

Results-The age at onset of nodal symptoms was earlier in the offspring (43 years (95\% confidence intervals (CI) 38 to 47$) v$ 61 (CI 58 to 65); mean difference 18 years (CI 13 to 22): $p<0.001$ ) as was large joint symptom onset (48 years (CI 41 to 55) $v 67$ (CI 61 to 73); mean difference 20 years (CI 13 to 27): $p<0.01$ ). A negative correlation existed between age of offspring symptom onset and parental age at conception. Fifteen $(50 \%)$ offspring had similar or more extensive disease than their parents.

Conclusions-These results suggest genetic anticipation occurs in NOA and if confirmed a search for trinucleotide repeats is warranted.

(Ann Rheum Dis 1998;57:524-526)
\end{abstract}

Genetic anticipation describes a tendency for certain diseases to occur at an earlier age in subsequent generations, with increasing severity in the offspring. A number of monogenic diseases including myotonic dystrophy, ${ }^{1}$ Huntington's disease ${ }^{2}$ and fragile $\mathrm{X}$ syndrome ${ }^{3}$ display this phenomenon. The biological basis of genetic anticipation is an increased number of trinucleotide repeat sequences close to or within the disease predisposing gene. Evidence exists for a direct correlation between the size of the expansion and the severity of the disease, and an inverse correlation with the age of onset. ${ }^{4}$ An inverse correlation also exists, in some disorders displaying anticipation, between the parental age of conception and the offspring age of disease onset. ${ }^{5}$ This correlation is greater with paternal than maternal age and may reflect ongoing mitosis throughout the lifetime of the paternal germ cells, with an increasing chance of unstable alleles expanding over time. Recently, features consistent with genetic anticipation have been described for genetically complex diseases such as bipolar affective disorder, ${ }^{6}$ schizophrenia, ${ }^{7}$ rheumatoid arthritis, ${ }^{8}$ and Crohn's disease. ${ }^{9}$

Nodal osteoarthritis (NOA), a subset of osteoarthritis, is characterised by polyarticular interphalangeal and thumb base OA, Heber- den and Bouchard node formation, a preponderance in women, and a clear genetic predisposition. NOA was first recognised as a discrete clinical entity by Kellegren and Moore $^{10}$ and various studies have shown that genetic factors play an important part. ${ }^{11-13}$ One study found that Heberdens and Bouchards nodes were present in $36 \%$ of the relatives of men with NOA and in $49 \%$ of the relatives of affected women in comparison to the expected frequencies in the general population of $17 \%$ and $26 \%$ respectively. ${ }^{12}$ Recently a classic twin study demonstrated a clear genetic influence on the development of NOA with a calculated score for heritability influence ranging from 39-65\%. ${ }^{14}$ Although no causative gene/s have been identified we have previously described a possible linkage to a region on the short arm of chromosome 2 containing three candidate genes in sib-pairs with NOA. ${ }^{15}$

As part of a larger study into the genetics of NOA we wished to determine whether there is evidence for genetic anticipation occurring in this disorder. Such evidence may explain the variable severity and age at onset reported for this disease and would suggest that a search for unstable trinucleotide repeat sequences in NOA might be worthwhile.

\section{Methods}

Approval for this study was obtained from the local research ethics committee. As part of a larger study collecting families and sib-pairs with NOA, multicase families with NOA were identified via unrelated community based studies, advertising campaigns, and rheumatology outpatient clinics. NOA was defined as the presence of Heberden's and/or Bouchard's nodes affecting at least two rays of either hand with or without large joint osteoarthritis (OA) involvement. Index cases were identified and the offspring or parent were interviewed and examined separately. Information regarding their date of birth, the age of symptom onset in their hands and large joints was ascertained. Symptom onset was defined as the occurrence of pain, stiffness or swelling in the affected joint. Clinical evidence of OA was confirmed by history and clinical examination. The number of nodes in each person was counted and graded on a scale $1-4$ according to size and position $(1=$ radial superolateral; $2=$ ulnar superolateral; 3 = radial and ulnar superolateral; 4 = posterior bar). Large joint OA was confirmed by clinical examination (restriction of passive range of movement $+/$ - crepitus or bony deformity) or a history of joint replacement.

Differences in the age of nodal and other joint symptom onset in the offspring and their parents were compared using a Student paired 
Table 1 Comparison of age of onset of nodal and large joint $O A$ symptoms between parent/offspring pairs with NOA

\begin{tabular}{lll}
\hline & Parents ( $n=30)$ & Offspring ( $n=30)$ \\
\hline Mean age at onset of nodal symptoms (y) (95\% CI) & $61(58$ to 65$)$ & $43(38 \text { to } 47)^{\star}$ \\
Age at onset of large joint OA (y) (95\% CI) & $67(58$ to 65) & $\begin{array}{l}48(41 \text { to } 55)^{\star} \\
\mathrm{n}=16\end{array}$ \\
\hline
\end{tabular}

*Statistically significantly (see text)

$t$ test. Correlation coefficients were calculated for age of symptom onset in the offspring with parental ages at offspring conception.

\section{Results}

Thirty parent/offspring pairs with NOA were identified. In 17 pairs the index case was the offspring. Fifteen index cases were identified through unrelated community based studies or advertising campaigns. Twenty eight parent/ offspring pairs were female/female; one was female/male and the other was male/male. Intraobserver reproducibility for the scoring of nodes was good ( $\kappa=0.9$ for distal interphalangeal and $\kappa=0.6$ for proximal interphalangeal nodes). The mean age at onset of nodal symptoms was significantly earlier in the offspring compared with the parents (43 years $(95 \%$ confidence intervals (CI) 38 to 47 ) $v 61$ years (CI 58 to 65); mean difference 18 years (CI 13 to 22$): p<0.001$ ) in the offspring/parent pairs. Clinical evidence of large joint OA was present in both offspring and parents in 16 offspring/ parent pairs and the mean age of symptom onset was earlier in the offspring (48 years (CI 41 to 55) $v 67$ years (CI 61 to 77); mean difference 20 years, (CI 13 to 27), p<0.01). These results are summarised in table 1 .

The mean duration of symptoms in the offspring was 8.4 years (range $2-12$ years) and despite their younger age the mean number of nodes was only marginally less than their parents (8 (range 2-17) v $11(3-18)$ ) as was the mean grade of node (or mean nodal total severity score) $(17(2-44) \cup 24(3-50))$ and mean number of large joints involved (1.6 $(0-9)$ v $2(0-8))$. Two (6\%) offspring had similar disease severity and $13(43 \%)$ had more severe and extensive disease as judged by a greater number and grade of nodes and/or a greater number of other joints involved. Of those 13 offspring with more severe disease than their parents nine had a greater number and grade of nodes than their parents while four had a greater number of large joints involved but a similar or fewer number and grade of nodes.

There was a significant negative correlation $(r=-0.38 ; \mathrm{p}<0.03)$ between the age of symptom onset in the offspring and parental age at conception. The offspring in the only paternal/ offspring pair had the earliest age of onset of symptoms (25 years) with the oldest age of parent at conception (40 years) When this paternal/offspring pair was eliminated from the analysis, and only maternal/offspring pairs were analysed, the correlation between age of symptom onset in the offspring and parental age at conception remained weakly negative $(-0.18)$ but not statistically significant.

\section{Discussion}

The concept of genetic anticipation has been debated from the early years of the century with myotonic dystrophy as the most striking example. ${ }^{16}$ Throughout most of this period there has been controversy as to whether the phenomenon resulted from observational and ascertainment biases or reflected a more fundamental mechanism. In 1992 a trinucleotide repeat (CTG) sequence was discovered at the $3^{\prime}$ non-coding region of the myotonic dystrophy gene, which is normally transmitted in a stable form from one generation to the next. The normal number of CTG copies in the population is between 6 and 30 . If an increase beyond 30 CTG copies occurs, an allele is produced that acts in an unstable form in transmission. ${ }^{1}$ If these expanded alleles are transmitted in germlines they appear capable of undergoing large expansions in early zygotic cell divisions. ${ }^{17-19}$ These expanded trinucleotide repeats are the underlying mutations that explain anticipation in a number of other neurological conditions. ${ }^{20}$ There also appears to be a direct correlation between the size of the expansion and the severity of the disease and an inverse correlation with the age of onset. ${ }^{41}$ These discoveries confirmed that genetic anticipation is indeed a true biological phenomenon secondary to specific as yet undefined molecular mechanisms.

In our study group there was evidence of a significantly younger age of disease onset (both nodal and large joint $\mathrm{OA}$ ) in the offspring and despite their younger age, 15 $(50 \%)$ had similar or more severe and extensive disease than their parents. As there is no universally agreed measure of "severity" of NOA we used the number and grade of nodes and the number of large joints involved clinically as a reproducible and quantifiable measure of disease severity.

There was also a significant negative correlation between the age of symptom onset in the offspring and parental age at conception. When the single paternal/offspring pair was eliminated from the analysis, the correlation between age of symptom onset in the offspring and parental age at conception remained weakly negative but was not statistically significant. This finding is in keeping with the phenomena of genetic anticipation as in some diseases in which unstable trinucleotide repeats have been demonstrated, older fathers tend to have children with a younger age of disease onset. ${ }^{5}$ This probably reflects ongoing mitosis throughout the lifetime of the paternal germ cells, with increasing chances of unstable premutant alleles expanding over time. Ova do not undergo further mitosis after the birth of the female and therefore increased mitosis in male germ cells should result in an increased mutation rate for fathers. There are, however, observations in monogenic disorders that suggest specific maternal transmission effects. For example, in fragile $\mathrm{X}$ syndrome expansion of the premutation occurs only through the maternal line. ${ }^{22}$ 
As NOA is predominantly a female disease, with relatively late disease expression, it will be difficult to confirm the influence of paternal age at conception on the age of onset in this disorder. Other possible explanations for the observations in this study include genomic imprinting or differential $\mathrm{X}$ chromosome inactivation ${ }^{23}$ and age related maternal in utero effects.

Studies of genetic anticipation are potentially subject to a number of biases. Firstly, ascertainment of pedigrees in which the parent develops disease late in life and the offspring develops disease early in life may lead to earlier reporting of symptoms in the offspring because of awareness and anxiety of familial disease. Secondly, pedigrees in which the parent develops early onset disease and the offspring shows late onset disease are less likely to be identified. Thirdly, symptom reporting is retrospective and may be influenced by other variables such as age, disease duration, levels of disability, anxiety, and depression. The presence of such biases occurring in this study group appear to be unlikely as; (1) 17 of the index cases were offspring with parents who were previously undiagnosed and had not sought medical attention; (2) 15 of the index cases were identified via unrelated community based projects or advertising campaigns; (3) no parent reported an earlier onset of symptoms than their offspring; and (4) the difference in symptom onset between offspring and their parents was in the order of two decades, which is unlikely to result from chance alone.

This study suggests genetic anticipation occurs in NOA. If confirmed, a search for expanded trinucleotide repeats is warranted to further elucidate the molecular basis of this common disorder.

This work was supported by the UK Arthritis and Rheumatism Council (Grant No. DO521). We are indebted to our research metrologists Sally Doherty and Desda Jordan and also Andrea metrologists Sally Doherty and

1 Brook JD, McCurrach ME, Harley HG, Buckler AJ, Church $\mathrm{D}$, Aburatani $\mathrm{H}$, et al. Molecular basis of myotonic D, Aburatani $\mathrm{H}$, et al. Molecular basis of myotonic dystrophy: expansion of a trinucleotide repeat (CTG) at the
3' end of a transcript encoding a protein kinase family 3' end of a transcript encoding
2 Huntington Disease Collaborative Research Group. A novel gene containing a trinucleotide repeat that is expanded and gene containing a trinucleotide repeat that is expanded and
unstable on Huntington's disease chromosomes. Cell 1993:7able on Hunti

3 Verkerk AJMH, Pieretti M, Sutcliffe JS, Fu YH, Kuhl DP, Pizzuti A, et al. Identification of a gene (FMR-1) containing a CGG repeat coincident with a break-point region exhibiting length variation in fragile X syndrome. Cell 1991;65: 905-14.

4 Harley HG, Rundle SA, Mac Millan JC, Myring J, Brook $\mathrm{JD}$, Crow $\mathrm{S}$, et al. Size of the unstable CTG repeat sequence in relation to phenotype and parental transmission in myotonic dystrophy. Am J Hum Genet 1993;52: sion in myoto $1164-743$.

5 Zheng CJ, ByersB, Moolgavkar SH. Allelic instability in mitosis: a unified model for dominant disorders. Proc Natl mitosis: a unified model for domina

6 McInnis MG, McMahon FJ, Chase GA, Simpson SG, Ross $\mathrm{CA}$, De Paulo JR. Anticipation in bipolar affective disorders. Am J Hum Genet 1993;53:385-90.

7 Basset AS, Honer WG. Evidence for anticipation in schizophrenia. Am J Hum Genet 1994;54:864-70.

8 Deighton C Heslop P, McDonagh J, Walker D, Thomson G. Does genetic anticipation occur in familial rheumatoid arthritis? Ann Rheum Dis 1994;53:833-5.

9 Polito II JM, Rees RC, Childs B, Mendeloff AI, Harris ML, Bayless TM. Preliminary evidence for genetic anticipation in Crohn's disease. Lancet 1996;347:798-800.

10 Kellgren JH, Moore R. Generalised osteoarthritis and Heberdens nodes. BMJ 1952;1:81-7.

11 Stecher RM, Hersch AH. Heberdens nodes the mechanism of inheritance in hypertrophic arthritis of the fingers. J Clin Invest 1994;23:699-704.

12 Kellegren JH, Lawrence JS, Bier F. Genetic factors in generalied osteoarthritis. Ann Rheum Dis 1963;22:237-55.

13 Harper P, Nuki G. Genetic factors in osteoarthritis. In: NukiG, ed. The aetiopathogenesis of osteoarthritis. Tunbridge Wells: Pitman, 1980:184-201.

14 Spector TD, Cicuttini F, Baker J, Loughlin JA, Hart DJ. Genetic influences on osteoarthritis in females: a twin study. BMJ 1996;312:940-3.

15 Wright GD, Hughes AE, Regan M, Doherty M. Association of two loci on chromosome $2 \mathrm{q}$ with nodal osteoarthritis. Ann Rheum Dis 1996;55:317-19.

16 Harper PS, Harley HG, Reardon W, Shaw JJ. Anticipation in myotonic dystrophy: New light on an old problem. Am J Hum Genet 1992;51:10-16.

17 Buxton J, Shelbourne P, Davies J, Jones C, Tongeren van T, Aslanidis C, et al. Detection of an unstable fragment of DNA specific to individuals with myotonic dystrophy. Nature 1992;355:547-8.

18 Harley HG, Brook JD, Rundle SA, Crow S, Reardon W, Buckler AJ, et al. Expansion of an unstable DNA region and phenotypic variation in myotonic dystrophy. Nature 1992;355:545-6.

19 Aslanidis C Jansen G, Amemiya C, Shutler G, Mahade van $M$, Tsilfidis $\mathrm{C}$, et al. Cloning of the essential myotonic dystrophy region and mapping of the putative defect. Nature 1992;355:545-6.

20 Maddox J. Triplet repeat genes raise questions. Nature 1994;368:685

21 Adams P, Falek A, Arnold J. Huntington disease in Georgia: age at onet. Am J Hum Genet 1988;43:695-704.

22 McConkie-Rosell A, Lachiewicz AM, Spiridigliozzi GA, Tarleton J, Schoenwald S, Phelan MC, et al. Evidence that Tarleton J, Schoenwald S, Phelan AC, et al. Evidence that phenotypic expression of the fragile $\mathrm{X}$ syndrome. Am J phenotypic expression of the

23 Gregerson PK. Discordance for autoimmunity in monozygotic twins. Are "identical" twins really identical? Arthritis Rheum 1993;36:1185-92. 